\title{
A Simple Assay to Screen Antimicrobial Compounds Potentiating the Activity of Current Antibiotics
}

\author{
Junaid Iqbal, ${ }^{1}$ Ruqaiyyah Siddiqui, ${ }^{1}$ Shahana Urooj Kazmi, ${ }^{2}$ and Naveed Ahmed Khan ${ }^{1}$ \\ ${ }^{1}$ Department of Biological and Biomedical Sciences, Aga Khan University, Stadium Road, Karachi 74800, Pakistan \\ ${ }^{2}$ Department of Microbiology, University of Karachi, Karachi 75270, Pakistan
}

Correspondence should be addressed to Naveed Ahmed Khan; naveed5438@gmail.com

Received 9 April 2013; Revised 29 May 2013; Accepted 4 June 2013

Academic Editor: Kuo-Chen Chou

Copyright (c) 2013 Junaid Iqbal et al. This is an open access article distributed under the Creative Commons Attribution License, which permits unrestricted use, distribution, and reproduction in any medium, provided the original work is properly cited.

\begin{abstract}
Antibiotic resistance continues to pose a significant problem in the management of bacterial infections, despite advances in antimicrobial chemotherapy and supportive care. Here, we suggest a simple, inexpensive, and easy-to-perform assay to screen antimicrobial compounds from natural products or synthetic chemical libraries for their potential to work in tandem with the available antibiotics against multiple drug-resistant bacteria. The aqueous extract of Juglans regia tree bark was tested against representative multiple drug-resistant bacteria in the aforementioned assay to determine whether it potentiates the activity of selected antibiotics. The aqueous extract of J. regia bark was added to Mueller-Hinton agar, followed by a lawn of multiple drug-resistant bacteria, Salmonella typhi or enteropathogenic E. coli. Next, filter paper discs impregnated with different classes of antibiotics were placed on the agar surface. Bacteria incubated with extract or antibiotics alone were used as controls. The results showed a significant increase $(>30 \%)$ in the zone of inhibition around the aztreonam, cefuroxime, and ampicillin discs compared with bacteria incubated with the antibiotics/extract alone. In conclusion, our assay is able to detect either synergistic or additive action of $J$. regia extract against multiple drug-resistant bacteria when tested with a range of antibiotics.
\end{abstract}

\section{Introduction}

Bacterial infections result in 17 million deaths worldwide annually, mostly in children and the elderly [1]. The morbidity and mortality associated with bacterial infections have remained significant, despite advances in antimicrobial chemotherapy. The situation has worsened with bacterial resistance increasing to available antibiotics. From the 1930s to 1960 s, twenty classes of antibiotics were marketed but in the last four decades only three new classes of antibiotics have been introduced [1-5]. Today, multiple drug-resistant bacteria such as methicillin-resistant Staphylococcus aureus (MRSA), vancomycin-resistant enterococci (VRE), Acinetobacter baumannii, Klebsiella pneumonia, Escherichia coli, and Pseudomonas aeruginosa pose a serious threat to human and animal health. There is also the emergence of bacterial pathogens with intrinsic resistance to antibiotics making the available antibiotics obsolete [2]. A feasible approach to counter such a crisis is to revive the use of available antibiotics against multiple drug-resistant bacteria by improving their efficacy. Here we suggest a simple, inexpensive and easy assay to perform screening of antimicrobial compounds from natural products [1] such as Juglans regia or synthetic chemical libraries for their potential to work in tandem with the available antibiotics against multiple drug-resistant bacteria. The basis of this assay is that the extract may enhance the killing effect of synthetic antibiotics by overcoming the resistant phenotype.

\section{Materials and Methods}

2.1. Bacterial Isolates. Two clinical isolates of Salmonella typhi and enteropathogenic E. coli were used in this study. E. coli isolate was derived from diarrheal stool sample and that of Salmonella typhi was obtained from the blood cultures of a typhoid patient. The clinical samples were plated on blood agar plates. All the bacterial isolates were characterized by colony morphology and biochemical testing and their identity confirmed by using API-20E system (bioMérieux). Both isolates were preserved in glycerol stocks. For both bacteria, 
antibiotic evaluation test was performed on Mueller-Hinton agar using Kirby-Bauer method as per Clinical Laboratory Standards Institute [6, 7]. Salmonella typhi was considered multiple drug resistant, if it exhibited growth on antibiotic disks containing ampicillin $10 \mu \mathrm{g}$ (breakpoint inhibition zone for ampicillin for $S$. typhi was considered $14 \mathrm{~mm}$ ), cefuroxime $30 \mu \mathrm{g}$ (breakpoint inhibition zone for cefuroxime for S. typhi was considered $18 \mathrm{~mm}$ ), and aztreonam $10 \mu \mathrm{g}$ (breakpoint inhibition zone was considered $24 \mathrm{~mm}$ ) as per EUCAST guidelines. Similarly, E. coli was considered multiple drug resistant, if it exhibited growth on antibiotic disks containing ampicillin $10 \mu \mathrm{g}$ (breakpoint inhibition zone for ampicillin for E. coli was considered $14 \mathrm{~mm}$ ), cefixime $5 \mu \mathrm{g}$ (breakpoint inhibition zone was considered $17 \mathrm{~mm}$ ), and aztreonam $10 \mu \mathrm{g}$ (breakpoint inhibition zone was considered $24 \mathrm{~mm}$ ).

2.2. Juglans regia Extract. The aqueous extract of J. regia (commonly known as Persian walnut or English walnut tree) bark was prepared by taking 10 gram bark and mixing it in $100 \mathrm{~mL}$ water overnight. Next day, the suspension was incubated in boiling water bath for two minutes. Subsequently, the mixture was cooled, centrifuged at $2500 \times \mathrm{g}$, and the supernatant was filtered through $0.2 \mu \mathrm{m}$ filter. The filtrate was evaporated using a refrigerated CentriVap Concentrator (Labconco Corp.) evaporator until dry and stored at $4^{\circ} \mathrm{C}$ until further use.

2.3. Simple Plating Assay. The proposed assay utilizes the modified version of the antibiotic disc diffusion assay ("Kirby-Bauer" assay) [6, 7]. The Mueller-Hinton agar plates were prepared containing aqueous extract of J. regia (final concentration of extract was $0.625 \mathrm{mg}$ per $\mathrm{mL}$ of agar). Next, $20 \mathrm{~mL}$ of the overnight grown culture of multiple drug-resistant bacteria, S. typhi and E. coli, was poured on Mueller-Hinton agar containing J. regia extract plates and left for $5 \mathrm{~min}$. Following this incubation, the excess bacterial cultures were aspirated and plates were left in the biosafety hood to dry. Next, filter paper discs impregnated with different antibiotics (aztreonam, cefuroxime, ampicillin, and cefixime) were placed on the agar surface and plates were incubated at $37^{\circ} \mathrm{C}$ overnight. Any change in the diameter of the inhibition zone was measured to determine bacterial sensitivity as per Clinical Laboratory Standards Institute [7]. Any increase in the diameter of the inhibition zone was investigated to determine whether a specific antibiotic plus J. regia extract has the potential to target the multiple drugresistant bacteria. For controls, S. typhi and E. coli were plated on Mueller-Hinton agar plates containing extract but without antibiotic discs. In some experiments, S. typhi and E. coli were plated on Mueller-Hinton agar plates without extract but with antibiotic discs.

\section{Results}

3.1. Aqueous Extract of J. regia Has Either a Synergistic Or Additive Action against Multidrug-Resistant (MDR) Bacteria When Tested with a Range of Antibiotics. When plated on Mueller-Hinton agar plates containing J. regia extract but without antibiotic discs, the growth of neither S. typhi nor E. coli was affected. However, in the presence of antibiotics, the results showed that the zone of inhibition for S. typhi around the aztreonam antibiotic disc increased from $20 \mathrm{~mm}$ in Mueller-Hinton agar plates to $29 \mathrm{~mm}$ in Mueller-Hinton agar containing $J$. regia extract plates. Thus, the addition of J. regia extract apparently renders once-resistant S. typhi strain sensitive to aztreonam again (breakpoint inhibition zone diameter of $\leq 24 \mathrm{~mm}$ was considered resistant) (Table 1). Similarly, the zone of inhibition around the ampicillin antibiotic disc increased from $13 \mathrm{~mm}$ in Mueller-Hinton agar plates to $19 \mathrm{~mm}$ in Mueller-Hinton agar containing J. regia extract plates (Table 1). Thus, the addition of J. regia extract apparently renders once-resistant $S$. typhi strain sensitive to ampicillin again (breakpoint inhibition zone diameter of $\leq 14 \mathrm{~mm}$ was considered resistant) (Table 1). However, J. regia had limited effect on the resistance of $S$. typhi strain to cefuroxime. The zone of inhibition around the cefuroxime antibiotic disc increased from $12.5 \mathrm{~mm}$ in Mueller-Hinton agar plates to $18 \mathrm{~mm}$ in Mueller-Hinton agar containing $J$. regia extract plates (breakpoint inhibition zone diameter of $\leq 18 \mathrm{~mm}$ was considered resistant) (Table 1 ).

The zone of inhibition for enteropathogenic E. coli around the ampicillin antibiotic disc increased from $13 \mathrm{~mm}$ in Mueller-Hinton agar plates to $18 \mathrm{~mm}$ containing J. regia extract plates. Thus, the addition of J. regia extract renders once-resistant $E$. coli strain sensitive to ampicillin again (breakpoint inhibition zone diameter of $\leq 14 \mathrm{~mm}$ was considered resistant) (Table 1). Similarly, the zone of inhibition around the cefixime antibiotic disc increased from $16 \mathrm{~mm}$ in Mueller-Hinton agar plates to $22 \mathrm{~mm}$ in Mueller-Hinton agar containing J. regia extract plates (Table 1 ). Thus, the addition of $J$. regia extract renders once-resistant $E$. coli strain sensitive to cefixime again (breakpoint inhibition zone diameter of $\leq 17 \mathrm{~mm}$ was considered resistant) (Table 1). However, J. regia extract had limited effect on the resistance of $E$. coli strain to aztreonam. The zone of inhibition around the aztreonam antibiotic disc increased from $14.5 \mathrm{~mm}$ in Mueller-Hinton agar plates to $21 \mathrm{~mm}$ in Mueller-Hinton agar containing $J$. regia extract plates (breakpoint inhibition zone diameter of $\leq 24 \mathrm{~mm}$ was considered resistant) (Table 1). Both E. coli and S. typhi lawns grew normally on plates added with J. regia's extract without antibiotics discs (data not shown).

\section{Discussion}

The basis of the proposed assay is that natural products with potential antibacterial activity overcome the resistance phenotype of multiple drug-resistant (MDR) bacteria and render them susceptible to the available antibiotics. The proposed assay has the potential to test the efficacy of various antibiotics on a single plate in an easy-to-use manner. In addition, the proposed assay is cost-effective and can be performed in a basic microbiology laboratory. Bacteria employ a variety of strategies to develop resistance against a single or group of antibiotics. These include drug-degrading and -modifying enzymes, reduced membrane permeability for drugs, expression of drug efflux pumps, overproduction 
TABLE 1: Juglans regia extract helped enhance the efficacy of synthetic antibiotics and overcame the resistant phenotype. Data are presented as the mean \pm standard error of three independent experiments.

\begin{tabular}{lccc}
\hline Antibiotic & $\begin{array}{c}\text { Zone of } \\
\text { inhibition, } \\
\text { mm (antibiotic } \\
\text { alone) }\end{array}$ & $\begin{array}{c}\text { Zone of } \\
\text { inhibition, mm } \\
\text { (antibiotic }+ \\
\text { Juglans regia } \\
\text { extract) }\end{array}$ & P value* \\
\hline $\begin{array}{l}\text { Aztreonam (MDR } \\
\text { Salmonella typhi) }\end{array}$ & $20 \pm 3.4$ & $29 \pm 6.2$ & $<0.01$ \\
$\begin{array}{l}\text { Cefuroxime (MDR } \\
\text { Salmonella typhi) }\end{array}$ & $12.5 \pm 2.2$ & $18 \pm 2.5$ & $<0.01$ \\
$\begin{array}{l}\text { Ampicillin (MDR } \\
\text { Salmonella typhi) }\end{array}$ & $13 \pm 2.5$ & $19 \pm 1.8$ & $<0.001$ \\
$\begin{array}{l}\text { Aztreonam (MDR } \\
\text { enteropathogenic E. coli) }\end{array}$ & $14.5 \pm 3.5$ & $21 \pm 5.3$ & $<0.01$ \\
$\begin{array}{l}\text { Cefixime (MDR } \\
\text { enteropathogenic E. coli) }\end{array}$ & $16 \pm 5.1$ & $22 \pm 4.7$ & $>0.05$ \\
$\begin{array}{l}\text { Ampicillin (MDR } \\
\text { enteropathogenic E. coli) }\end{array}$ & $13 \pm 0.9$ & $18 \pm 3.1$ & $>0.01$ \\
\hline
\end{tabular}

${ }^{*} P$ values were calculated using one-tailed paired $t$-test.

and alteration of drug targets, and bypassing the inhibitory pathway [2]. Thus, strategies (or use of natural products) that can block the aforementioned drug resistance mechanisms will allow the clinical use of currently available antibiotics. This is not a novel concept and several studies have reported the use of multiple compounds to produce synergistic or additive effects against microbial pathogens $[3,6,8,9]$. One such example is coamoxiclav, a combination of amoxicillin and clavulanic acid. This combination is also effective against amoxicillin-resistant bacteria because clavulanic acid is an inhibitor of $\beta$-lactamase, an enzyme which degrades $\beta$ lactam drugs including amoxicillin. Other similar combinations which are in clinical use include ampicillin/sulbactam and piperacillin/tazobactam [10]. The findings reported here suggest that J. regia extract can extend the clinical use of available antibiotics. This is consistent with previous findings which suggest that $J$. regia bark extract has either synergistic or additive action against Gram-negative bacteria [11]. However, the mechanisms of action remain unknown, and future studies are needed to identify the substance(s) present in the $J$. regia extract responsible for the synergy with $\beta$-lactams. Notably, J. regia is commonly used in some countries as a toothbrush, and it has been suggested that brushing teeth with this bark may improve oral hygiene, prevent plaque and caries formation, and reduce the incidence of gingival and periodontal infections [12]. Although there is no toxicity data available for $J$. regia, recent findings suggest that $J$. regia extract treatment $(150 \mathrm{mg} / \mathrm{kg})$ resulted in protective restoration of decreased antioxidants in a murine urotoxicity model [13], albeit at considerable lower concentrations compared with the present study.

It is instructive to point out that many molecular biosystems and biomedical systems belong to the multilabel systems in which each of the constituent molecules possesses one or more than one function or feature and hence needs one or more than one label to indicate its attribute(s), as observed in recent antimicrobial peptides research [14]. Antimicrobial peptides (AMPs) belong to the class of well-studied multifunctional molecules. They are part of innate immune mechanisms, found in almost all cellular organisms. Many AMPs have been shown to possess a broad spectrum of activities against viruses, bacteria, fungi, protists, parasites, and surprisingly, even against tumor cells [15]. Mostly, AMPs are cationic peptides and work through interfering with cell membrane integrity or enter into the target cell and inhibit vital cellular processes like DNA replication, transcription, translation, and metabolic pathways [15]. Other important features of AMPs include their low toxicity to mammalian cells and slow rate of emergence of bacterial resistance against them in comparison with the available antibiotics [16]. Recently, a number of powerful computational tools have been developed to predict or design AMPs bearing one or more activities [17, 18]. All of the above features have made AMPs a promising candidate for future therapeutics [15]. However, as a first step, here we have tested the single-label system. Many studies have indicated that cheminformatics [19], mutagenesis [20], molecular docking [21, 22], predicting drug-target interaction [23], and bioinformatics [24, 25] can timely provide very useful information and insights for drug development and hence are widely welcomed by the scientific community. The present study attempted to develop a novel method to screen potential antimicrobials using plate assay, hoping that the new method can become a useful tool for drug development.

Overall, our findings provide support that J. regia extract enhances the efficacy of tested antibiotics against multiple drug-resistant bacteria. The proposed assay can easily be used to screen a wide range of natural products and compound libraries. Our assay has an advantage of screening a pure compound or natural product against five to eight different antibiotics on the same plate. Because of its cost effectiveness and simplicity, this assay could be used by labs which have just basic microbiology setup and limited funding and allow them to contribute in the discovery of potential antimicrobials that target drug-resistant pathways in multiple drug-resistant bacteria, the mechanisms of which will be explored in further studies.

\section{Conflict of Interests}

The authors declare (1) no conflict of interests for the submitted work; (2) no financial relationships with commercial entities that might have an interest in the submitted work; (3) no spouses, partners, or children with relationships with commercial entities that might have an interest in the submitted work; and (4) no financial interest that may be relevant to the submitted work.

\section{Funding}

This work was supported by the Aga Khan University, Pakistan. 


\section{References}

[1] M. S. Butler and A. D. Buss, "Natural products-the future scaffolds for novel antibiotics?" Biochemical Pharmacology, vol. 71, no. 7, pp. 919-929, 2006.

[2] F. C. Tenover, "Mechanisms of antimicrobial resistance in bacteria," The American Journal of Medicine, vol. 119, no. 6, pp. S3-S10, 2006.

[3] J. H. Powers, "Antimicrobial drug development-the past, the present, and the future," Clinical Microbiology and Infection, vol. 10, no. 4, pp. 23-31, 2004.

[4] A. R. Coates, G. Halls, and Y. Hu, "Novel classes of antibiotics or more of the same?" The British Journal of Pharmacology, vol. 163, no. 1, pp. 184-194, 2011.

[5] V. M. Dcosta, C. E. King, L. Kalan et al., "Antibiotic resistance is ancient," Nature, vol. 477, no. 7365, pp. 457-461, 2011.

[6] P. L. Ho, K. H. Chow, K. Y. Yuen, W. S. Ng, and P. Y. Chau, "Comparison of a novel, inhibitor-potentiated disc-diffusion test with other methods for the detection of extended-spectrum $\beta$-lactamases in Escherichia coli and Klebsiella pneumoniae," Journal of Antimicrobial Chemotherapy, vol. 42, no. 1, pp. 49$54,1998$.

[7] National Committee for Clinical Laboratory Standards, "Performance standards for antimicrobial susceptibility testing," in NCCLS Approved Standard M100-S14, NCCLS, Wayne, Pa, USA, 2004.

[8] S. Donadio, S. Maffioli, P. Monciardini, M. Sosio, and D. Jabes, "Antibiotic discovery in the twenty-first century: current trends and future perspectives," Journal of Antibiotics, vol. 63, no. 8, pp. 423-430, 2010.

[9] M. Pestel, E. Martin, C. Aucouturier, J. F. Lemeland, and F. Caron, "In vitro interactions between different $\beta$-lactam antibiotics and fosfomycin against bloodstream isolates of enterococci," Antimicrobial Agents and Chemotherapy, vol. 39, no. 10, pp. 2341-2344, 1995.

[10] S. Rochon-Edouard, M. Pestel-Caron, J. F. Lemeland, and F. Caron, "In vitro synergistic effects of double and triple combinations of $\beta$-lactams, vancomycin, and netilmicin against methicillin-resistant Staphylococcus aureus strains," Antimicrobial Agents and Chemotherapy, vol. 44, no. 11, pp. 3055-3060, 2000.

[11] A. M. Alkhawajah, "Studies on the antimicrobial activity of Juglans regia," The American Journal of Chinese Medicine, vol. 25, no. 2, pp. 175-180, 1997.

[12] P. Nancy, M. Manasi, and A. Varghese, "Antiplaque activity of Juglans regia L. and characterization of Juglone from Juglans regia L," The American Journal of Biochemistry and Biotechnology, vol. 7, no. 1, pp. 29-31, 2011.

[13] K. Bhatia, S. Rahman, M. Ali, and S. Raisuddin, "In vitro antioxidant activity of Juglans regia L. bark extract and its protective effect on cyclophosphamide-induced urotoxicity in mice," Redox Report, vol. 11, no. 6, pp. 273-279, 2006.

[14] K. C. Chou, "Some remarks on predicting multi-label attributes in molecular biosystems," Molecular BioSystems, vol. 9, no. 6, pp. 1092-1100, 2013.

[15] B. M. Peters, M. E. Shirtliff, and M. A. Jabra-Rizk, "Antimicrobial peptides: primeval molecules or future drugs?" PLoS Pathogens, vol. 6, no. 10, Article ID e1001067, 2010.

[16] G. G. Perron, M. Zasloff, and G. Bell, "Experimental evolution of resistance to an antimicrobial peptide," Proceedings of the Royal Society B, vol. 273, no. 1583, pp. 251-256, 2006.
[17] P. Wang, L. Hu, G. Liu et al., "Prediction of antimicrobial peptides based on sequence alignment and feature selection methods," PLoS ONE, vol. 6, no. 4, Article ID e18476, 2011.

[18] X. Xiao, P. Wang, W. Z. Lin, J. H. Jia, and K. C. Chou, "iAMP-2L: a two-level multi-label classifier for identifying antimicrobial peptides and their functional types," Analytical Biochemistry, vol. 436, no. 2, pp. 168-177, 2013.

[19] J. Xu and A. Hagler, "Chemoinformatics and drug discovery," Molecules, vol. 7, no. 8, pp. 566-600, 2002.

[20] M. Congreve, C. W. Murray, and T. L. Blundell, "Keynote review: structural biology and drug discovery," Drug Discovery Today, vol. 10, no. 13, pp. 895-907, 2005.

[21] X. Y. Meng, H. X. Zhang, M. Mezei, and M. Cui, "Molecular docking: a powerful approach for structure-based drug discovery," Current Computer-Aided Drug Design, vol. 7, no. 2, pp. 146157, 2011.

[22] J. L. Stark and R. Powers, "Application of NMR and molecular docking in structure-based drug discovery," Topics in Current Chemistry, vol. 326, pp. 1-34, 2012.

[23] F. Cheng, C. Liu, J. Jiang et al., "Prediction of drug-target interactions and drug repositioning via network-based inference," PLOS Computational Biology, vol. 8, no. 5, Article ID e1002503, 2012.

[24] N. S. Buchan, D. K. Rajpal, Y. Webster et al., "The role of translational bioinformatics in drug discovery," Drug Discovery Today, vol. 16, no. 9-10, pp. 426-434, 2011.

[25] A. J. Butte and S. Ito, "Translational bioinformatics: data-driven drug discovery and development," Clinical Pharmacology and Therapeutics, vol. 91, no. 6, pp. 949-952, 2011. 

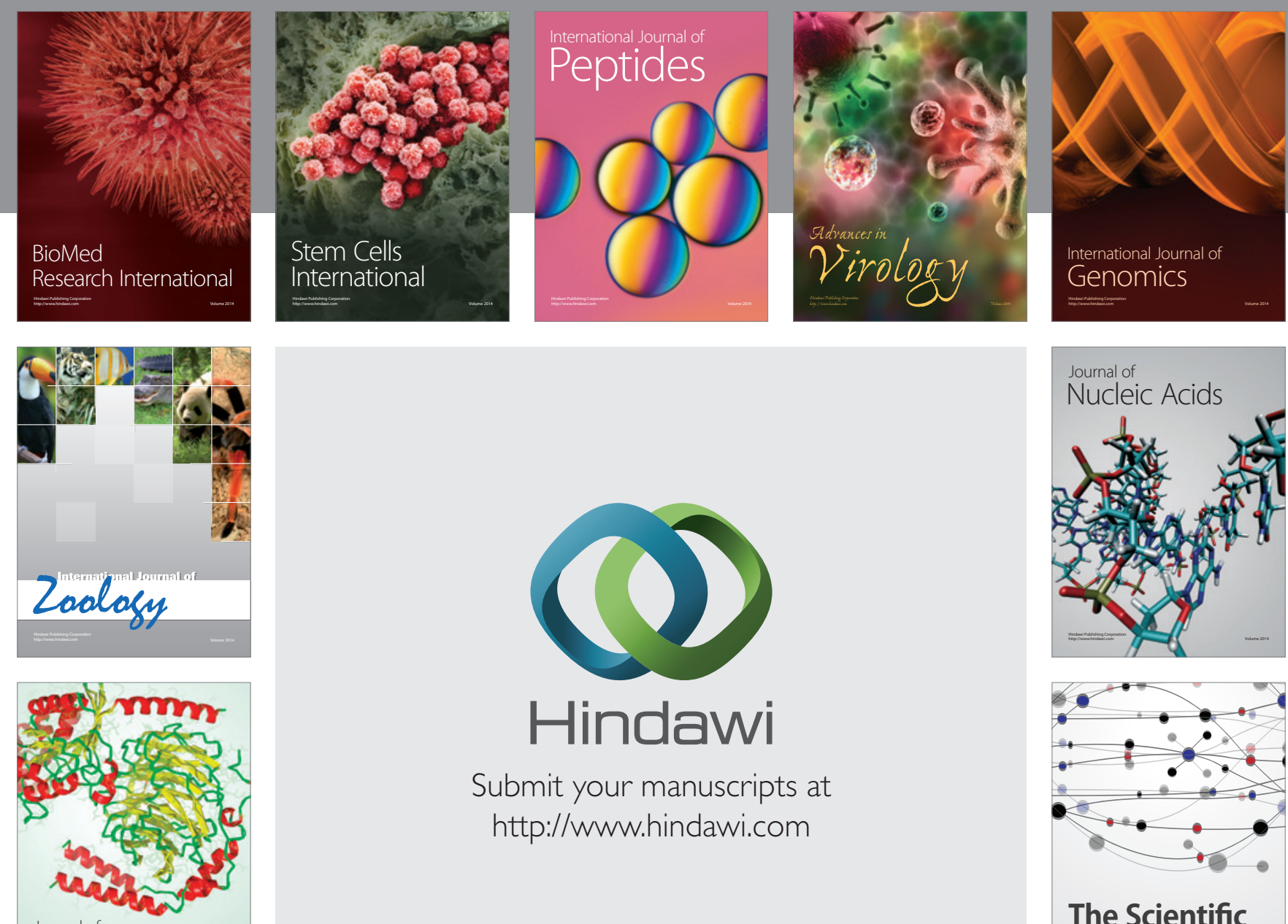

Submit your manuscripts at

http://www.hindawi.com

Journal of
Signal Transduction
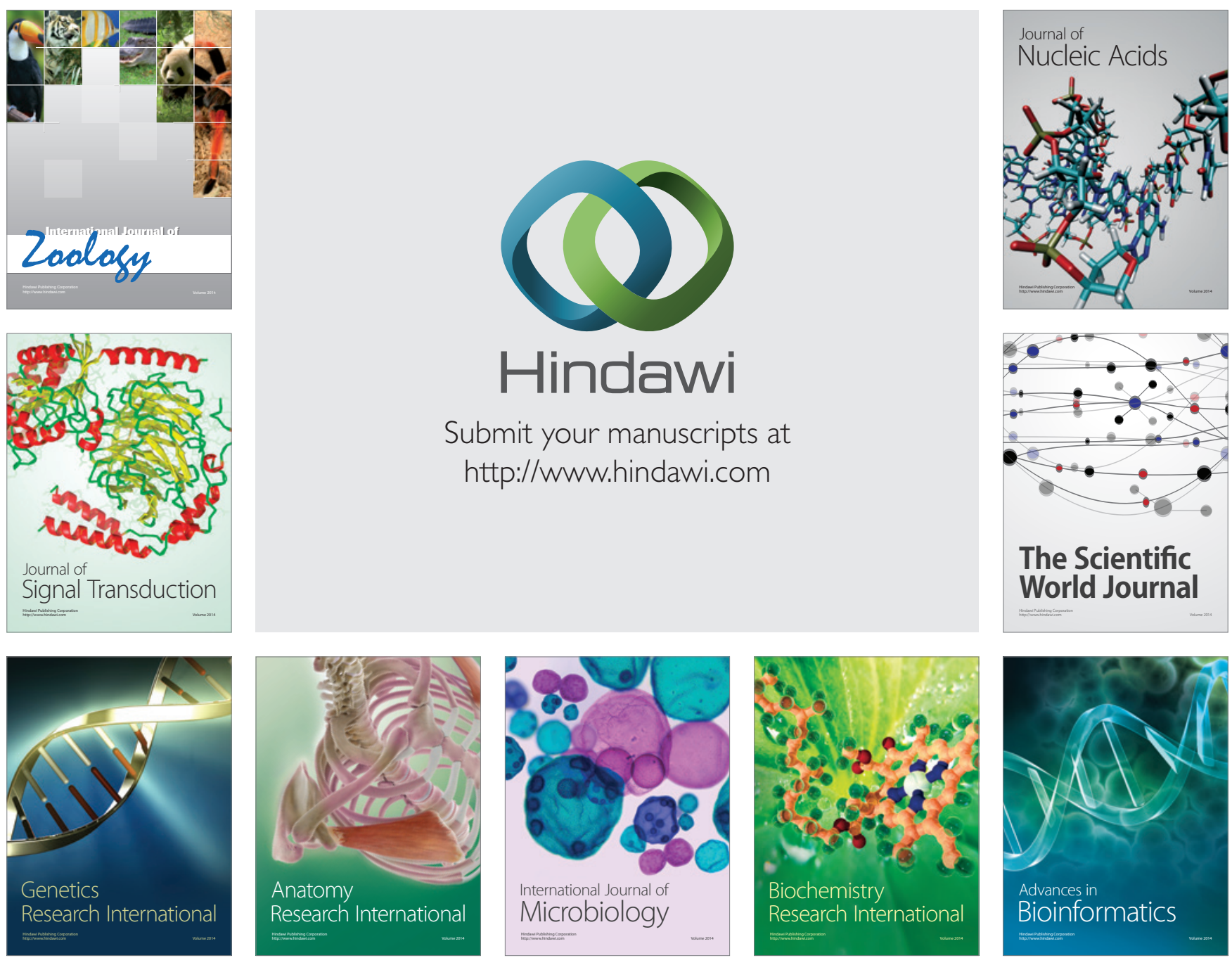

The Scientific World Journal
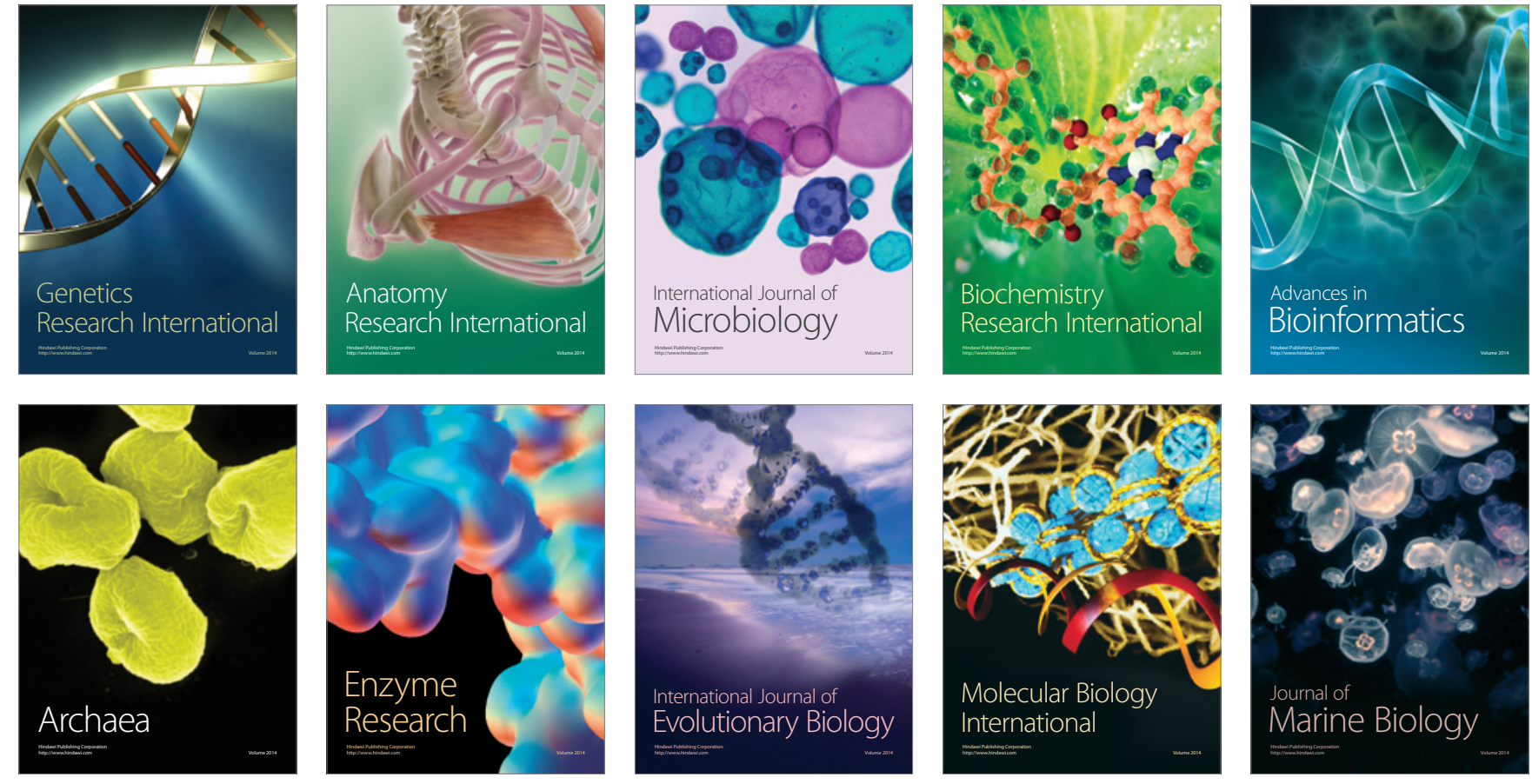